Questions de communication

$24 \mid 2013$

Renouvellement des mises en scène télévisuelles de la politique

\title{
Quel renouvellement des mises en scène télévisuelles de la politique?
}

Pierre Leroux et Philippe Riutort

\section{OpenEdition}

\section{Journals}

Édition électronique

URL : http://journals.openedition.org/questionsdecommunication/8644

DOI : 10.4000/questionsdecommunication. 8644

ISSN : 2259-8901

Éditeur

Presses universitaires de Lorraine

Édition imprimée

Date de publication : 31 décembre 2013

Pagination : 7-18

ISBN : 978-2-8143-0182-5

ISSN : $1633-5961$

Référence électronique

Pierre Leroux et Philippe Riutort, « Quel renouvellement des mises en scène télévisuelles de la politique? ", Questions de communication [En ligne], 24 | 2013, mis en ligne le 01 février 2014, consulté le 30 avril 2019. URL : http://journals.openedition.org/questionsdecommunication/8644 ; DOI : 10.4000/questionsdecommunication.8644 
PIERRE LEROUX

Centre de recherche sur l'action politique en Europe Institut d'études politiques de Rennes Université catholique de l'Ouest/L'Université Nantes Angers Le Mans

F-49000

pierre.leroux@uco.fr

PHILIPPE RIUTORT

Groupe d'analyse politique Université Paris Ouest Nanterre La Défense

F-92000

riutortp@yahoo.fr

\section{QUEL RENOUVELLEMENT DES MISES EN SCÈNE TÉLÉVISUELLES DE LA POLITIQUE ?}

1 de très rares et brèves exceptions près, jusqu'à la fin des années 90, la représentation des personnalités politiques s'est organisée sous l'égide des journalistes et des rédactions. La politique est restée le domaine réservé d'un petit nombre de professionnels reconnus partageant avec leurs interlocuteurs politiques la plupart des cadres de « vision et de division » (Boltanski, Bourdieu, 1976) de la politique, autrement dit une même idée de la représentation médiatique de la politique se référant, globalement, à un idéal d'échange centré sur la présentation d'arguments par les principaux acteurs de la démocratie représentative face à des contradicteurs et des questionneurs (opposants politiques et/ou journalistes, puis, plus récemment, citoyens ordinaires encadrés par des journalistes). Déjà amorcée, cette domination du 
logos (Lochard, Soulages, 2003) est encore plus nettement délaissée avec, à la fin des années 90, l'apparition régulière de professionnels de la politique au sein des premières émissions « conversationnelles »!

Affirmant dès l'origine leur volonté de désacralisation de la politique, ces émissions entendent intégrer les personnalités politiques à des programmes visant spécifiquement les publics a priori les moins intéressés par la politique. Pour appréhender ces nouveaux cadres de réception du personnel politique et les éventuelles transformations de la parole politique, les premières analyses se sont surtout focalisées sur les nombreux éléments qui distinguaient ces émissions des émissions politiques classiques qui les ont précédées en soulignant, notamment (et comparativement), leur faible portée politique 2 . À l'origine, certains éléments plaident en faveur de cette thèse : l'intégration d'invités politiques en faible proportion comparativement aux personnalités du spectacle, une tendance avérée à une apparente dépolitisation du discours qui ne permettrait pas de leur attribuer une portée politique, le faible écho médiatique des discours politiques tenus dans ces programmes (Leroux, Riutort, 20I I) qui pourrait permettre de conclure à leur relative vacuité politique. Dans un état antérieur du rapport émissions politiques/divertissements, cette perspective apparaissait relativement pertinente même si, depuis les années 80 , l'hybridation des programmes avait déjà largement redéfini le périmètre des émissions politiques (en particulier par la présence d'invités extérieurs au champ politique) $)^{3}$. Mais les évolutions des émissions conversationnelles ont rendu en grande partie caduques certaines des distinctions. D'emblée, les promoteurs des émissions de divertissement se sont situés dans une perspective concurrentielle vis-à-vis des journalistes tandis que beaucoup d'indices témoignent aujourd'hui d'un certain succès des animateurs

' La locution « émissions conversationnelles » désigne les émissions reprenant les codes de la conversation ordinaire dans la forme (interruptions, changement de sujets, interactions multiples) comme dans le fond (faible hiérarchie des intervenants, valorisation des qualités des réparties, des traits d'humour, etc.), distinctes des émissions politiques conçues pour l'exposé d'arguments recourant aux usages traditionnels de la rhétorique en politique. Dans sa contribution au dossier, Jeffrey P. Jones souligne aussi cet aspect (pour les talk shows des chaînes américaines) qui associe conversation et échanges interpersonnels avec le spectacle médiatisé. Toutefois, il s'agit d'une caractéristique générale et dominante des émissions qui connait de nombreuses variantes d'une formule à l'autre. Dans ces émissions, la parole vise moins à « donner sa vision du monde politique comme fondée, fondée dans l'objectivité » (Bourdieu, 1995), qu'à s'insérer dans une dynamique d'échanges rapides où la place du discours politique n'est pas définie a priori comme dominante (contrairement aux émissions définies par les programmateurs comme « politiques »).

2 Aurélien Le Foulgoc (2007) considère les émissions conversationnelles comme des émissions « horscadre » ; Érik Neveu (2003) pointe l'évacuation du politique, Éric Darras (2005) relève l'importance de l'effet de reprise des déclarations dans les émissions politiques, et leur quasi-inexistence dans celles de divertissement.

3 Parmi les programmes ressortissant d'une hybridation politique/divertissement, on relèvera Sept sur Sept (TFI), Les Absents ont toujours tort (La Cinq), L'Hebdo (Canal +) et une grande partie des émissions politiques des années 2000 comme 100 minutes pour convaincre (France 2) ou À vous de juger (France 2) comportant respectivement 39 et 45 \% d'invités autres que politiques (Le Foulgoc, 2007), c'est-à-dire aussi bien des célébrités que des experts ou, éventuellement, des anonymes. 
dans cette redéfinition de statuts. En effet, ces derniers ont réussi à faire apparaître certaines approches alternatives de la représentation politique, débarrassées du projet politique que les journalistes mettent souvent en avant (éclairer les choix démocratiques) pour affirmer un objectif spectaculaire (au sens où il s'agit d'intégrer les politiques et la politique au spectacle télévisuel ${ }^{4}$ ). En raison de son histoire politique (Bourdon, 1994 ; Olivesi, 1998), la télévision française était le dernier média à avoir pu préserver le traitement de la politique de toute atteinte à sa majesté, les autres (radio, presse écrite, etc.) ayant largement affaibli les distinctions, que ce soit par les modes de traitement, les approches ou les centres d'intérêt ${ }^{5}$. Ainsi les émissions conversationnelles prennent-elles place dans un mouvement général d'aggiornamento du traitement médiatique de la politique. Elles ne sauraient avoir à elles seules transformé le rapport de la télévision à la politique, même si elles ont contribué à une évolution des cadres télévisuels de mise en scène de la politique en prenant acte de la désacralisation de la politique dans l'espace public médiatisé. En témoigne la progressive participation de journalistes reconnus aux émissions de divertissement (aujourd'hui généralisées) qui a sans doute joué un rôle décisif quant à leur reconnaissance, sur les plans symbolique et pratique. En conséquence, la frontière entre divertissements et émissions politiques classiques s'en est trouvée plus atténuée tandis que tous les types d'émission peuvent prétendre - avec des succès variés - contribuer à la formation du débat public.

\section{Caractériser les divertissements politiques}

En France, il faut attendre la fin des années 90 pour voir programmées sur les chaînes privées et publiques les premières émissions de divertissement accueillant des personnalités politiques. Même si le personnel politique n'a jamais - même du temps du monopole public de la télévision - été uniquement présent dans les émissions politiques ${ }^{6}$, elles introduisent une forme de rupture dans la représentation télévisuelle de la politique. On peut les définir à partir de trois caractéristiques. D'abord, ces programmes sont rattachés à la direction des programmes (et non aux rédactions) et sont donc souvent produits et présentés par des animateurs (plutôt que par des journalistes). Ainsi ces

\footnotetext{
${ }^{4}$ Le fait que les trois premières émissions de divertissement apparues à l'antenne (Le Vrai journal, Vivement dimanche et Tout le monde en parle) soient des émissions enregistrées puis montées atteste de la visée spectaculaire : il s'agit d'accélérer le rythme des échanges et d'alléger le discours politique des « temps morts », c'est-à-dire le plus souvent, des éléments qui pourraient les rapprocher des thématiques et du discours des émissions politiques classiques.

5 Par exemple, témoignent de l'atténuation des distinctions entre visées politiques et spectaculaires les tranches matinales d'information à la radio, l'emblématique Paris Match pour les magazines de presse écrite, mais aussi les évolutions plus générales qui touchent ces médias auxquelles s'ajoutent les modalités spécifiques et multiples utilisées pour aborder la politique sur l'internet.

${ }^{6}$ Des Dossier de l'écran (Office de radiotélévision française, puis Antenne 2) dans les années 60-70, aux émissions comme Apostrophes (Antenne 2) ou Droit de réponse (TFI) dans les années 80, on a pu voir que la présence des personnalités politiques ne se limitait pas aux émissions politiques.
} 
émissions s'autorisent-elles l'affranchissement ostentatoire des règles et codes qui ont auparavant gouverné la relation de la télévision avec la politique (Mouchon, 1998). Ainsi l'élite professionnelle des animateurs - profession devenue un acteur essentiel à la faveur du basculement général en direction de la télévision commerciale au milieu des années 80 (Leroux, Riutort, 2006) valorise-t-elle symboliquement sa position : l'accès à la sphère politique constituait le dernier terrain ( $p$ )réservé des journalistes. Le franchissement de cette frontière offre des perspectives nouvelles, notamment une prétention à apporter une contribution civique au débat démocratique, chose inimaginable dans un état antérieur de la télévision (Chalvon-Demersay, Pasquier, 1990). Deuxième caractéristique, ces émissions intègrent le personnel politique de façon explicite et régulière dans la construction même du programme. Les politiques deviennent un ingrédient permanent de l'émission tout en demeurant, certes, minoritaires (à la différence des émissions politiques), mais en occupant progressivement une place incontestable. Enfin, troisième caractéristique, ces émissions ne sont pas conçues spécifiquement pour les politiques. Ces derniers sont tenus de prendre place au sein d'un dispositif dont la logique est étrangère à l'univers politique et aux débats sur les affaires publiques et emprunte aux codes du divertissement, rodés depuis longtemps pour recevoir les invités du spectacle. Ainsi la parole politique trouve-t-elle dans ces dispositifs conversationnels une place très différente de celle qu'elle pouvait avoir dans les émissions politiques ou les journaux télévisés. Sa suprématie ne va plus de soi et elle se trouve confrontée, comme jamais auparavant, à un parasitage permanent, pensé comme un outil de déstabilisation, plus ou moins violent et explicite selon les formules, celles-ci étant tributaires des contraintes spécifiques découlant de l'horaire de programmation, du public potentiel et du format. Les rôles dévolu au public (forcément réactif et enthousiaste, et demandeur de moments spectaculaires ${ }^{8}$ ), à l'animateur principal et à ses acolytes (chroniqueurs et snipers connu pour leur capacité de réactivité et leurs formules déstabilisantes), aux différentes séquences qui rythment l'émission (qui, dans les faits, viennent contrecarrer les formes conventionnelles du discours politique) et, éventuellement - lorsque l'émission n'est pas diffusée en direct -, le montage ultérieur concourent à la construction d'un spectacle sans cesse renouvelé, évitant plus efficacement tunnels, exposés abscons et développements argumentés que ne pouvaient se l'autoriser les journalistes à l'égard du personnel politique dans les formules classiques de débats et d'échanges?

\footnotetext{
7 Sur le concept de dispositif issu des travaux de M. Foucault, voir Noël Nel (1998) qui revient sur cette filiation et son importation pour l'analyse de la télévision. Voir aussi Violaine Appel, Hélène Boulanger et Luc Massou (2010).

8 Le conditionnement préalable du public par le chauffeur de salle l'incite à demander des moments spectaculaires.

9 Cependant, les journalistes sont toujours intervenus pour fluidifier le débat à travers des consignes pour limiter notamment la multiplication de données chiffrées et d'exposés trop longs ou trop techniques.
} 
Sous des formes variées, des animateurs proposent donc les premiers divertissements intégrant le personnel politique. Le Vrai Journal (Canal +, 1996-2006) comporte une interview d'une dizaine de minutes (à partir d'un entretien réel de 45 à 60 minutes) d'une personnalité politique dans une émission comportant (vrais) reportages et parodies ; Vivement dimanche (France 2, 1998-) ${ }^{10}$ décline auprès des politiques la formule du dévoilement de l'intimité publique d'un invité principal rodée auparavant avec des personnalités du spectacle (parcours biographique, goûts, « face cachée » des personnalités) et Tout le monde en parle (France 2, 1998-2006) adopte rapidement la formule de l'entretien en plateau d'une personnalité politique autour d'une table parmi des invités issus du monde du spectacle. Ce dispositif sert de patron - avec de nombreuses variantes - pour des émissions comme On ne peut pas plaire à tout le monde (France 3, 2000-2006), T'empêches tout le monde de dormir (M6, 2006-2008), Vendredi et Samedi pétantes (Canal +, 2003-2006), Le Grand Journal (Canal +, 2004-) et On n'est pas couché (France 2, 2006-) qui contribueront à banaliser ce cadrage de la politique.

\section{L'heure des bilans?}

Dès l'origine, la nouveauté, en France, de ces mises en scène a suscité de nombreuses interrogations et protestations qui posaient la question des transformations des cadres de représentation de la politique « saisie par le divertissement » (CoulombGully M., Tournier M., 2001 ; Réseaux, 2003). Ces émissions conduisaient-elles à la marginalisation, voire au déclin définitif des formes traditionnelles de mise en scène télévisuelle de la politique animées par les journalistes? La question était posée et ce point de vue a été défendu par les journalistes politiques et certains observateurs de la télévision dans une perspective alarmiste : « L'américanisation de la vie politique », le « triomphe du spectaculaire », le « sacre de l'infotainment » étaient évoqués, le mélange politique/divertissement ayant une responsabilité décisive dans une « crise » générale de la démocratie. En les situant dans un mouvement d'ensemble (Sartori, 1989) et en tenant compte des reconfigurations plus larges de la représentation de la politique, il est permis de relativiser la rupture introduite par ces émissions, pour mieux en situer les véritables ressorts et enjeux. En France, il revient à Érik Neveu (1995) d'avoir très tôt posé des jalons permettant de situer dans une continuité l'émergence de nouveau cadres d'expression des professionnels de la politique : les « impasses » (Neveu, 1995), voire « l'échec » (Coulomb-Gully, Tournier, 200I) du spectacle télévisuel de la parole politique ; dans les émissions politiques devait logiquement conduire à des formes de renouvellement et le modèle des États-Unis pouvait logiquement

\footnotetext{
10 Nous désignerons sous ce titre l'ensemble de l'émission comportant en réalité deux parties: Vivement dimanche (l'après-midi) et Vivement dimanche prochain, le soir avant le journal télévisé. Voir dans ce dossier l'article de B. Lafon sur les contenus de cette émission.
} 
inspirer les professionnels français' '. Le même auteur situe aussi les analyses critiques de ces transformations dans le cadre des recherches à l'étranger permettant implicitement d'identifier les spécificités du terrain français... mais aussi le fond commun de certaines oppositions idéologiques qui sous-tendent les analyses et les débats ${ }^{12}$. Sur le plan des discours et de leur transformation, la révolution de la « néo-télévision » (Casseti, Odin, 1990), celle du rapport à l'intimité (Mehl, 1996) et, plus spécifiquement, de la parole politique (Lochard, Soulages, 2003) soulignent aussi des continuités : le discours politique s'insère dans les transformations plus générales de la parole à la télévision et cet élargissement de la focale permet de prendre la véritable mesure de l'originalité de la mise en scène des politiques au sein des divertissements.

La référence à ces travaux permet de situer le propos du dossier qui se fixe pour objectif de questionner l'articulation politique/télévision à travers ces formes de représentation télévisuelle. En d'autres termes, prenant acte de la pérennisation des émissions conversationnelles (elles existent depuis déjà une quinzaine d'années en France), on peut se demander ce qui a permis à celles-ci de perdurer et de définitivement prendre place au sein des mises en scène médiatiques de la politique. Pour le personnel politique de premier plan (quasiment seul convié dans les grands rendez-vous télévisuels), si la participation aux émissions politiques classiques a correspondu - et correspond encore - à un devoir démocratique et à une croyance dans la capacité de ces tribunes à intéresser et convaincre le citoyen-téléspectateur, l'intérêt de la participation aux émissions de divertissement et leur utilité politique ne sont pas allées de soi, du moins, au-delà des cas particuliers et des objectifs généraux (notoriété, rencontre avec un public élargi) $)^{13}$ souvent mis en avant. Mais ces objectifs généraux ne sont pas d'emblée partagés par l'ensemble du personnel politique. Bien plus, la prise de risque inhérente aux formes mêmes de ces émissions, nécessitant des compétences pour partie moins mobilisées dans l'exercice quotidien du métier politique, pouvait rendre problématique le succès, sur le long terme de ces « nouvelles »

"Les émissions « forum » et plus généralement la participation des profanes dans les émissions politiques ont pu apparaître à un moment comme une issue à l'« épuisement » de la parole politique. Sur ce point, voir É. Darras (1999), Sébastien Rouquette (2001, 2002) et P. Lefébure (2005).

12 Dans le dossier, notre propos n'étant pas de prendre parti sur l'intérêt démocratique de l'exposition du politique dans les divertissements, nous renvoyons sur ce point spécifique à la synthèse des débats et des analyses anglo-saxonnes présentée par É. Neveu (200I, 20I2) et l'échange entre Kees Brants et É. Neveu (2003) résume l'opposition de deux regards, à propos de formes diverses, de contextes différents (les Pays-Bas pour l'un, la France pour l'autre), alors que ces formes n'ont encore que quelques années d'existence. Sur ce point, voir aussi Liesbet Van Zoonen (2004).

13 Sur l'intérêt de la participation à des émissions de divertissement dans le but d'accrôtre sa notoriété et de rencontrer un public élargi, voir Apolline de Malherbe (2007). D'ailleurs, les données de cette auteure montrent que, bien avant l'existence des premières émissions conversationnelles, quelques personnalités politiques ont participé à de nombreux programmes de toute nature (et continueront par la suite). Ainsi J. Lang - ministre de la Culture (1981-1986), de la Culture et de la Communication (1988-1992), puis de l'Éducation nationale et de la Culture (1992-1993) sous la présidence de François Mitterrand - participet-il à 33 émissions avant le lancement des programmes conversationnels - il apparait à Tout le monde en parle dès 1999 et participe à 75 émissions de divertissement sur la période 1986-2006 (ibid. : 223-228). 
tribunes. Pourtant, aujourd'hui, alors que l'effet de nouveauté s'est largement émoussé, un premier constat peut être dressé : ces émissions se sont banalisées, elles se sont installées dans l'espace télévisuel comme dans la vie politique : en recevant la plupart des acteurs de premier plan, et à travers l'écho qui leur est donné, elles participent largement à la mise en évidence de la politique, rendue visible et accessible à un large public. Comme un effet de leur institutionnalisation ${ }^{14}$ en tant que tribune politique d'un genre spécifique, leur présence dans la campagne de l'élection présidentielle française de 2012 s'est réalisée sans débats de fond et sans mise en cause des chaînes qui les programmaient. Bien plus, et à la différence de ce qu'on avait pu observer auparavant, notamment lors de l'élection présidentielle de 2007 (soit qu'elles aient été déprogrammées, soit qu'elles durent se limiter dans leurs choix d'invités), les talk shows - selon la désignation générique - recevant les politiques ${ }^{15}$ se sont pleinement inscrits dans cette campagne. Ils ont reçu les candidats et leurs représentants qui se sont soumis aux règles de représentation du champ politique édictées par les instances de contrôle de l'audiovisuel' ${ }^{16}$. Pour comprendre ce succès, on doit interroger la dynamique d'évolution de ces émissions. En effet, on ne saurait décrire cette institutionnalisation de façon linéaire et c'est en cela que l'étude de l'articulation politique/télévision paraît pertinente. Les formules ont profondément évolué et, au-delà des changements de programmes (seule Vivement dimanche a survécu jusqu'à aujourd'hui et Le Grand Journal a près de dix ans d'existence), les émissions ont sans cesse été conduites à repenser leur relation à la politique. Bien que la formule conversationnelle semble s'être imposée ${ }^{17}$, la mécanique interne des émissions n'a plus grand-chose à voir avec ce qu'elle a pu être au commencement. La banalisation de la réception des politiques s'est faite au prix de multiples ajustements et concessions à l'univers politique, innovations et emprunts à d'autres modalités de relation à la politique, qui, voués à l'échec ou au succès d'audience, témoignent de la plasticité d'un genre autant que de sa capacité d'adaptation. Si l'on veut mesurer d'éventuels effets de ces émissions sur la représentation politique, il ne faut sans doute pas l'analyser comme un

\footnotetext{
14 Par le terme « institutionnalisation », nous désignons le fait que, par-delà la disparition des émissions ou leur transformation, la place de ce type d'émission dans les programmes semble définitivement acquise.

${ }^{15}$ Aujourd'hui, les talk shows recevant les politiques sont essentiellement quatre émissions : Le Grand Journal et Le Petit Journal (Canal +, émissions distinctes depuis la rentrée 20II), Salut les Terriens (Canal +) et On n'est pas couché (France 2).

${ }_{16}$ En France, la campagne dans les médias audiovisuels est réglée par différents temps se référant à des principes d'équité puis d'égalité parfaite des temps de parole. Sur ce point, voir le site du Conseil supérieur de l'audiovisuel (CSA). Par ailleurs, le cahier des charges des chaines historiques impose la présence de programmes recevant des interlocuteurs politiques sans précision concernant la forme même des émissions. Ainsi TFI, chaîne privée, se voit-elle obligée par le CSA de diffuser « régulièrement des magazines d'information politique à des heures de grande écoute ». Accès : http://www.csa.fr/Television/Les-chaines-de-television/Les-chaines-hertziennes-terrestres/ Les-chaines-nationales-gratuites. Consulté le 01//0/13.

${ }_{17}$ La formule conversationnelle met l'invité politique en relation avec des personnalités du spectacle (souvent grand public) et évite la relation duelle (autrefois présente dans Le Vrai Journal et dans En aparte sur Canal +).
} 
triomphe général du divertissement dans la mise en scène de la politique (ce qui ne permet guère de conclure à une importation du modèle en vigueur aux États-Unis où ces émissions ont depuis longtemps acquis une place majeure, comparable à d'autres types d'émissions politiques classiques). Les formules plus classiques d'émissions politiques n'ont pas disparu. Elles ont trouvé une place nouvelle, certes sans commune mesure avec celle qu'occupaient les grands rendez-vous politiques d'antan, davantage conséquence de la démultiplication de l'offre de programmes ${ }^{18}$ que de la concurrence des divertissements accueillant le personnel politique. Ainsi les émissions conversationnelles s'inscrivent-elles comme une offre supplémentaire de mise en scène de la politique, susceptible de réunir un public relativement large sans entamer les performances d'audience des rendez-vous plus classiques soumis, de ce point de vue, aux aléas de la vie politique et à ses temps forts ${ }^{19}$. La spécificité des émissions conversationnelles d'aujourd'hui doit donc être examinée.

L'articulation politique/divertissement est interrogée dans le dossier à partir de plusieurs entrées. Les auteurs du premier article reviennent sur la question de la participation du personnel politique aux divertissements. Prenant acte du fait que l'activité politique en tant que champ existe à travers un certain nombre de positions, d'intérêts, de capitaux propres aux individus, Pierre Leroux et Philippe Riutort posent la question de la relation des politiques aux scènes du divertissement, pour l'inscrire dans la dynamique d'évolution des formes et des discours. Partant de la question de «l'invitation » implicitement lancée aux politiques par les animateurs producteurs des divertissements, les auteurs cherchent à comprendre comment se réalise concrètement au fil du temps, mais de façon différenciée en fonction des programmes, la participation des principaux leaders politiques aux différentes formules. Comment les promoteurs de ces programmes réussissent-ils à imposer de nouveaux modes de représentation de la politique? De quelles tensions témoignent les difficultés plus ou moins grandes de pénétration de l'univers politique? Les auteurs montrent que c'est en étudiant la nature du rapport de force entre ces programmes et leurs participants que l'on peut comprendre leur progressive légitimation.

Ce questionnement général est prolongé par Benoit Lafon qui étudie les intérêts et stratégies communicationnelles croisés des acteurs médiatiques et politiques à travers le cas de l'invitation du leader du Nouveau Parti anticapitaliste (NPA) Olivier Besancenot

\footnotetext{
${ }^{18}$ Avec la multiplication des chaînes thématiques accessibles au plus grand nombre (télévision numérique terrestre, distribution par les fournisseurs d'accès à internet), largement alimentées par des échanges politiques (chaînes d'information en continu, chaînes parlementaires), le volume global d'offre de programmes intégrant des participants politiques apparait beaucoup plus dispersé qu'il ne l'était au cours de la période précédente (Le Foulgoc, 2007 ; Eschstruth, 2007).

19 Sans surprise, les émissions politiques classiques sur les grandes chaînes enregistrent leurs records d'audience dès lors qu'elles s'inscrivent dans une compétition électorale disputée (c'est le cas de la présidentielle) et qu'elles reçoivent les représentants les mieux placés dans la compétition. En temps ordinaire, les audiences sont en général dépendantes de la personnalité reçue, de l'attente provoquée par l'émission, de son rapport à l'agenda politique et, enfin, de la concurrence des autres programmes.
} 
à l'émission Vivement dimanche de Michel Drucker en mai 2008. Considérée comme emblématique des formes nouvelles de communication politique (et de la construction d'une identité politique fortement axée sur des traits de la personnalité), on prête parfois à cette émission une capacité incomparable de valorisation des acteurs politiques et des performances traduisibles en bénéfices électorau $x^{20}$. Le faible nombre de politiques invités chaque année dans ce programme dominical (à l'opposé des vedettes du spectacle autour desquelles l'émission est construite) et la sélectivité qui en résulte ont contribué à sa renommée. Elle a réussi à s'imposer peu de temps après la réception des premiers interlocuteurs politiques comme un lieu disputé d'apparition du personnel politique et est considérée par les commentateurs comme une version apaisée de divertissement (il suffirait pour le politique de s'y montrer tel qu'il est). Benoit Lafon montre que, plutôt que ces mérites et pouvoirs supposés et au-delà de l'apparente simplicité des contenus, le passage à l'émission emprunte à de nombreux registres (pas seulement à celui du divertissement) et relève d'autres enjeux. Vivement dimanche donne lieu à une intense activité journalistique et est analysée par l'auteur comme un investissement, sur les plans politique et journalistique, dans et sur le marché politique. L'écho dont bénéficie le passage d'un professionnel de la politique à l'émission, nouvelle forme de « pièges à herméneutes » (Champagne, 1990) est ainsi - au moins tout autant que l'audience - un indice de son poids politique et apporte sa contribution à la notabilisation du personnel politique.

Patrick Amey analyse ensuite le discours spécifique et la mise en scène de la politique d'une autre émission conversationnelle emblématique, Le Grand Journal (Canal +). En 1984, l'émission prend la suite d'une autre en plateau (Nulle Part ailleurs) qui ne traitait qu'indirectement de la politique (principalement à travers la parodie de journal télévisé, Les Guignols de l'info). Le Grand Journal aménage progressivement des espaces de réception du politique et accède au personnel politique de premier plan, réussissant ainsi à devenir la référence quasi unique en matière d'infotainment à la française. Tout en s'inspirant de modèles étrangers, Le Grand Journal initie à travers un cadrage spécifique de la politique préservant apparemment le politique de la contiguité avec l'univers du spectacle, tout en l'inscrivant dans un dispositif commun global. Comme le montre l'étude (sur un corpus d'émissions diffusées en 2009), c'est le cadrage de la parole politique par le dispositif qui construit des cadres d'interprétation des actions et de la personnalité politique. L'auteur témoigne d'une période charnière de l'émission alors que les contenus humoristiques les plus attentatoires au politique ont disparu et que ce cadrage est conduit à prendre une nouvelle ampleur. L'évolution ultérieure de l'émission souligne la pertinence de l'analyse du chercheur : l'autonomisation du Petit Journal (à la rentrée 20I I) et la valorisation de « La petite question » (posée en plateau) confirme le succès de l'approche du Grand Journal sur le plan public, bien sûr, mais tout autant auprès du personnel politique comme lieu « incontournable » d'une forme de représentation dont Patrick Amey analyse l'ambiguité.

\footnotetext{
20 Selon le publicitaire J. Séguéla dans le documentaire La communication politique sous la Cinquième République (Gaillard, 2005, France 5), « aller faire le beau chez Michel Drucker [...] c'est un passage obligé si on veut être président des Français ».
} 
L'étude réalisée par Frédérick Bastien et David Dumouchel porte sur la version québécoise de Tout le monde en parle (Radio-Canada) - qui reprend les éléments du dispositif de l'émission française de Thierry Ardisson - présentée par Guy A. Lepage, comparée ici au Téléjournal. Elle constitue un prolongement pertinent du cas français et pose la question de la spécificité du traitement de la politique dans les divertissements. L'observation porte sur une période de campagne électorale. Partant de l'hypothèse d'une différenciation importante entre les types de questionnement des interviews politiques du journal télévisé et celles de Tout le monde en parle, les chercheurs en arrive au constat d'une absence de différences significatives. Les indicateurs retenus pour évaluer la rigueur respective des questionnements (types de question) et les sujets abordés (enjeux, stratégie, vie privée, personnalité) vont dans le même sens. Les pratiques journalistes/animateurs se différencient peu et le spectre traditionnel de sujets (y compris les enjeux pour le divertissement et la vie privée pour les journalistes) semble pouvoir invalider l'hypothèse d'un partage des rôles entre le divertissement et le travail journalistique.

Le dernier article permet d'introduire le cas emblématique des États-Unis où la place des talk shows, quantitativement plus importante qu'en France, est censée avoir fait figure de modèle et, le plus souvent, de repoussoir. Le texte de Jeffery $P$. Jones présente le grand intérêt de resituer toute la singularité de la situation des États-Unis où la présence déjà ancienne des chaînes d'information en continu, l'institutionnalisation d'émissions de satire politique conviant des invités politiques et l'influence des talk shows de l'après-midi dessinent un paysage fort différent de celui de la France. Ainsi semblerait-il que les talk shows américains, loin d'être homologues aux français, seraient davantage « politiques » (à la manière de ceux de Fox News érigés en véritable tribune politique), « divertissants » (au sens où le divertissement y rencontre beaucoup moins de résistances) et « populaires » (puisque les talk shows de l'après-midi sont spécifiquement destinés aux femmes issues des catégories populaires, encore une différence notable avec les émissions françaises du même type).

\section{Conclusion}

Les analyses proposées dans le dossier couvrent un spectre qui, sans être exhaustif, est suffisamment large pour tirer quelques conclusions sur le traitement de la politique dans le divertissement dans son ensemble. Elles attirent l'attention sur trois phénomènes : I) une dynamique d'évolution des cadrages de la politique qui ne se limite pas aux divertissements ; 2) une différenciation moindre que par le passé des approches de la politique élaborées par les journalistes et les animateurs ; 3 ) un repositionnement global de la télévision à l'égard de la représentation du politique. Nul doute que ces mouvements de fond conduiront, dans les années à venir, à des évolutions de formes, des discours et du rapport des médias à la représentation de la politique. 


\section{Références}

AppelV., Boulanger H., Massou L., dirs, 20 I 0, Les dispositifs d'information et de communication. Concepts, usages et objets, Bruxelles, De Boeck.

Boltanski L., Bourdieu P., 1976, « La production de l'idéologie dominante », Actes de la recherche en sciences sociales, 2/3, pp. 4-73.

Bourdieu P., 1995, « Champ politique, champ des sciences sociales, champ journalistique. Cours du Collège de France prononcé le 14 nov. 1995 à l'université Lumière Lyon 2 », Cahiers de recherche, 15.

Bourdon J., 1994, Haute fidélité. Pouvoir et télévision. 1935-1994, Paris, Éd. Le Seuil.

Brants K., 2003, « De l'art de rendre la politique populaire. Ou "qui a peur de l'infotainment?" »», Réseaux, I 18, pp. |35-166.

Casseti F., Odin R., 1990, « De la paléo à la néo-télévision. Approche sémio-pragmatique », Communication, 5I, pp. 9-26.

Chalvon-Demersay S., Pasquier D., 1990, Drôles de stars. La télévision des animateurs, Paris, Aubier.

Champagne P., 1990, Faire l'opinion. Le nouveau jeu politique, Paris, Éd. de Minuit.

Coulomb-Gully M., Tournier M., 200I, « La politique à l'écran : l'échec ? », Mots. Les langages du politique, 67, pp. 3-7.

Darras É., 1999, «Télévision et démocratisation. La "télévision forum" en France et aux États-Unis », pp. 6I-84, in : François B., Neveu É., dirs, Espaces publics mosaïques, Rennes, Presses universitaires de Rennes.

— 2005, « Permanences et mutations des émissions politiques en France », Recherches en communication, 24, pp. 109- 128.

Eschstruth I., 2007, « La politique et le pluralisme à la télévision à l'épreuve des chiffres du CSA ». Accès : http://www.acrimed.org/article2647.html. Consulté le 16/09//3.

Le Foulgoc A., 2007, Les représentations politiques reconfigurées par le divertissement à la télévision française. Des programmes pris dans des stratégies et des temporalités, thèse en sciences de l'information et de la communication, université Panthéon-Assas.

Lefébure P., 2005, Quand les citoyens discutent le lien représentatif, thèse en science politique, Institut d'études politiques de Paris.

Leroux P., Riutort P., 2006, «L La consécration de l'animateur.Appréciation d'un métier et affirmation d'une position : les métamorphoses de Thierry Ardisson », Réseaux, 139, pp. 219-248.

— 20I I, « Les émissions de divertissement: de nouveaux lieux de valorisation des petites phrases », Communication \& langages, | 68, pp. 69-80.

Lochard G., Soulages J.-C., 2003, « La parole politique à la télévision. Du logos à l'ethos », Réseaux, I 18, pp. 65-94.

Malherbe A. de, 2007, Politiques recherchent audimat désespérément, Paris, A. Michel.

Mehl D., 1996, La télévision de l'intimité, Paris, Éd. Le Seuil.

Mouchon J., 1998, La politique sous l'influence des médias, Paris, Éd. L'Harmattan. 
Nel N., 1998, « Les dispositifs télévisuels », pp. 59-73, in : Bourdon J., Jost F., éds, Penser la télévision, Paris, Nathan.

Neveu É., 1995, « Les émissions politiques à la télévision. Les années quatre-vingt ou les impasses du spectacle politique », Hermès, 17- | 8, pp. 145-162.

— 200 I, « Une crise de la parole politique à la télévision. Échos d'un débat anglophone », Mots. Les langages du politique, 67. pp. 8-27.

— 2003, « De l'art (et du coût) d'éviter la politique. La démocratie du talk-show version française (Ardisson, Drucker, Fogiel) », Réseaux, I | 8, pp. 95- 134.

— coord., 2003b, « La politique saisie par le divertissement », Réseaux, I 18.

— 2012, « Prosaïser le politique ou politiser le prosaïque », in : Amey P., Leroux P., dirs, 2012, L'échange politique à la télévision. Interviews, débats, et divertissements politiques, Paris, Éd. L'Harmattan.

Olivesi S., 1998, Histoire politique de la télévision, Paris, Éd. L'Harmattan.

Rouquette S., 200 I, L'impopulaire télévision populaire. Logiques sociales, professionnelles et normatives des palabres télévisées (1958-2000), Paris, Éd. L'Harmattan.

- 2002, Vie et mort des débats télévisés, Paris/Bruxelles, Ina/De Boeck.

Sartori G., 1989, «Video-Power », Government and Opposition, I, vol. 24, pp. 39-53.

Van Zoonen L., 2004, Entertaining The Citizen: When Politics and Popular Culture Converge, Lanham, Rowman \& Littlefield. 